\title{
Differential localization of laminin chains in bovine follicles
}

\author{
I. L. van Wezel, H. F. Rodgers and R. J. Rodgers* \\ Department of Medicine, Flinders University of South Australia, Bedford Park, South Australia 5042, \\ Australia
}

The composition of a basal lamina markedly affects its ability to filter material and affects the fate of adjacent epithelial cells. Therefore, basal laminae differ in composition with tissue development, and between different tissues in the body. Laminins are a component of basal laminae and consist of one $\alpha$, one $\beta$ and one $\gamma$ chain, of which there are at least five, three and two isoforms, respectively. This is the first study to immunolocalize a range of these individual laminin chains $(\alpha 1, \alpha 2, \beta 1, \beta 2, \gamma 1)$ in ovarian follicles. Frozen sections of bovine ovaries $(n=6)$ were immunostained using specific antisera to laminin chains and factor VIII-related antigen (to identify endothelial cells). Secondary antisera were labelled with one of two different fluorochromes (DTAF and $\mathrm{Cy} 3$ ), and dual localization of laminin chains and factor VIII-related antigen was performed. The $\alpha 1, \beta 2$ and $\gamma 1$ chains were consistently localized to the follicular basal lamina in all healthy follicles. Staining was less intense in the atretic antral follicles. Conversely, $\alpha 2$ and $\beta 1$ were rarely present in the follicular basal laminae of healthy antral follicles. Two of nine healthy antral follicles observed stained weakly for $\alpha 2$ in their basal lamina, and $\beta 1$ was present at low concentrations in growing preantral follicles. In atretic antral follicles, the follicular basal lamina stained positively for $\alpha 1, \alpha 2$, and $\beta 2$ but no $\beta 1$ was detected and the $\gamma 1$ staining was less intense than in healthy follicles. Antisera to Englebreth Holm-Swarm tumour laminin stained basal laminae of all follicles. In the theca of antral follicles, $\beta 1$ and $\beta 2$ chains were both present in the vasculature. Staining for the $\gamma 1$ chain was present in the thecal vasculature and generally throughout the theca of healthy and atretic antral follicles. Therefore, the composition of the follicular basal lamina alters during development and atresia, and potentially plays a role in the changing identity of the granulosa cells and the accumulation of antral follicular fluid.

\section{Introduction}

Basal laminae are specialized sheets of extracellular matrix that separate epithelial cell layers from underlying mesenchyme in organs throughout the body, including the ovary. They influence epithelial cell migration, proliferation and differentiation, and can selectively retard the through-passage of material. Basal laminae are a lattice-type network of collagen IV intertwined with a network of laminin. This structure is stabilized by the binding of entactin to the collagen and laminin, and by low-affinity interactions between collagen IV and laminin (Yurchenco and Schittny, 1990; Paulsson, 1992). Fibronectin, heparan sulfate proteoglycans (HSPGs) and other molecules are associated with the collagen IV-laminin backbone. It is significant that basal laminae in different regions of the body differ in the ratio of all these components. Furthermore, each "component' is in fact a class of several components. Thus, each collagen IV molecule is composed of three $\alpha$ chains, but six different types of $\alpha$ chain have been discovered to date, and any combination of these might be present (Hay, 1991; Zhou et al., 1994). Similarly, each laminin molecule is composed of

*Correspondence.

Received 5 June 1997. one $\alpha$ ( $A$ in the old nomenclature), one $\beta$ (B1 in the old nomenclature) and one $\gamma$ (B2 in the old nomenclature) chain (Burgeson et al., 1994), yet five different $\alpha$ chains, three $\beta$ chains and two $\gamma$ chains have been discovered. Owing to alternative splicing of mRNA, at least 20 different isoforms of fibronectin exist. The unique composition of each basal lamina is considered to contribute to its specific functional properties (Engvall, 1993).

Numerous studies in vitro have shown that cell morphology is altered according to the type of extracellular matrix component on which the cells are cultured (Watt, 1986). Thus, alterations to the basal lamina composition will affect the fate of the associated cells. The composition of basal laminae also affects their ability to selectively filter materials. For example, in normal neonatal mice, laminin $\beta 1$ is replaced by $\beta 2$ in the kidney glomerular basement membrane as the kidney develops. However, mice with a null mutation in the laminin $\beta 2$ gene continued with $\beta 1$, but then failed to retard the throughpassage of plasma proteins despite having a structurally intact glomerular basement membrane; these mice died of proteinuria within one month of birth (Noakes et al., 1995). In the ovary, the membrana granulosa of each ovarian follicle is enveloped by a follicular basal lamina, which separates it from the surrounding stromal elements in primordial follicles (van Wezel 
and Rodgers, 1996) or theca in antral follicles (Gosden $e t$ al., 1988; Luck, 1994). The follicular basal lamina is believed to play a role in influencing granulosa cell proliferation and differentiation (Amsterdam et al., 1989; Richardson et al., 1992; Luck, 1994). In addition, in healthy follicles, it excludes capillaries, white blood cells and nerve processes from the granulosa compartment until ovulation, at which time it is degraded. It probably also has a role retarding entry of larger molecular weight plasma proteins and molecules, for example low density lipoproteins (LDL), into the follicular antrum (Andersen et al., 1976). Conversely, it may trap in the follicular fluid large molecules, for example some proteoglycans that are synthesized by granulosa cells and oocytes.

The bovine follicle is estimated to double in surface area nineteen times during development, implying that continual remodelling of the follicular basal lamina occurs (van Wezel and Rodgers, 1996). The composition of the follicular basal lamina is hypothesized to alter during follicular development, particularly at the time follicular fluid accumulates to form an antrum, and during follicular atresia. Immunolocalization studies have demonstrated the presence of collagen IV (Bagavandoss et al., 1983; Kaneko et al., 1984; Palotie et al., 1984), laminin (Wordinger et al., 1983; Bagavandoss et al., 1983; Palotie et al., 1984; Leu et al., 1986; Christiane et al., 1988; YoshinagaHirabayashi et al., 1990; Leardkamolkarn and Abrahamson, 1992; Fröjdman et al., 1995), and fibronectin (Bagavandoss et al., 1983; Yoshimura et al., 1991; Figueiredo et al., 1995) in the follicular basal laminae of antral follicles. However, none of these studies has sought to differentiate between the different isoforms of any of these components, except one study which compared the localization of $\alpha I$ with that of $\beta I-\gamma I$ laminin in the ovary of the mouse fetus (Fröjdman et al., 1995). Other studies using western and northern blotting identified the expression of a few of the subtypes of collagen and laminin (Zhao and Luck, 1995; Iivanainen et al., 1995) but did not specifically localize these components to the follicular basal lamina; this is important since there are other basal laminae in follicles, such as those of the vasculature.

The laminin components in bovine follicles were immunolocalized using antibodies specific to the $\alpha 1, \alpha I, \beta 1, \beta 2$ or $\gamma 1$ chains of laminin to identify a range of individual laminin chains present in the follicular basal lamina.

\section{Materials and Methods}

\section{Tissues}

One ovary was obtained from each of six nonpregnant cows within 20 min of slaughter at a local abattoir. A slice of up to $5 \mathrm{~mm}$ was cut through the centre of the ovaries and they were immediately immersed in Tissue-Tek OCT Compound (Miles Inc, Elkhart, IN) and snap-frozen; these blocks were stored at $-70^{\circ} \mathrm{C}$. Tissue sections $(10 \mu \mathrm{m})$ were cut using a CM1800 Leica cryostat, collected on glass slides freshly treated with poly-L-lysine (Sigma Chemical Co., St. Louis, MO) and stored at $-20^{\circ} \mathrm{C}$ until use. Sections from each ovary were also fixed in acetone for $30 \mathrm{~min}$ and stained with haematoxylin and eosin by standard methods to facilitate assessment of follicular morphology. At least one section from each ovary was used for the immunolocalization of each laminin chain, and as a negative control.

\section{Classification of follicle health}

The term 'preantral' includes primordial follicles with one layer of flattened or flattened and cuboidal cells (van Wezel and Rodgers, 1996) and growing follicles with variable numbers of granulosa cell layers but without a visible antrum. On the basis of our previous observations using well preserved material (Faddy and Gosden, 1995), it would be expected that a negligible number of preantral follicles would be atretic. In the present study, even though the morphology of preantral follicles in frozen sections was only sufficient to identify changes taking place during moderate to severe atresia, most follicles appeared to be healthy. In antral follicles, a progression of marked changes to the membrana granulosa takes place during atresia (Rajakoski, 1960; Roy and Terranova, 1988). Therefore, the health of these antral follicles was classified according to the integrity of the membrana granulosa, and the presence or absence of cellular debris in the antrum. Antral follicles were classified as (1) 'healthy' if their membrana granulosa was intact and uniform in width, and if there was little or no cellular debris; or (2) 'atretic' if the membrana granulosa was not uniform in width, giving it a tattered appearance, or if there was a large amount of cellular debris. Follicles at more advanced stages of regression, such as those lacking granulosa cells and follicular scars, were not considered in this study.

\section{Antibodies}

Individual laminin chains were immunolocalized using rabbit polyclonal antisera raised against mouse recombinant laminin $\alpha 1$ chain (no. 317; Durbeej et al., 1997; 1/50-1/100 dilution), or against human $\alpha 2$ chain (no. 321 in preliminary studies, no. M4 in comprehensive studies; donated by M. Paulsson; 1/50 dilution). Antiserum no. 321 has been described by Paulsson and Saladin (1989). Antiserum no.M4 was raised by immunizing a rabbit with the $300 \mathrm{kDa} \alpha 2$ band from a preparative gel, after purification of human placental laminin as described by Lindblom et al. (1994). This antiserum was found to be specific for $\alpha 2$ when tested by immunoblot analyses (M. Paulsson, personal communication), and in our preliminary studies no. 321 and no. M4 produced equivalent staining patterns. Mouse monoclonal antibodies were those raised against human laminin $\beta 1$ chain (Cat. no. A004; Gibco BRL, Gaithersberg, MD; $1 / 500$ dilution), bovine laminin $\beta 2$ chain (no. C4, 1/100 dilution; Sanes and Chiu, 1983; Hunter et al., 1989), or human laminin $\gamma 1$ chain (Cat. no. A005; Gibco; $1 / 500$ dilution). In addition, rabbit polyclonal antiserum (Cat. no. L9393; Sigma Chemical Co., St Louis, MO; 1/100 dilution) raised against laminin-1 isolated from Englebreth Holm-Swarm (EHS) tumours and, therefore, detecting $\alpha I, \beta I$ and $\gamma 1$ chains, was used.

Non-laminin antibodies used in this study were rabbit anti-human von Willebrand factor (factor VIII-related antigen) IgG (Cat. no. F-3520; Sigma Chemical Co.; 1/100 dilution), for 
detection of endothelial cells (van Wezel and Rodgers, 1996), and mouse monoclonal anti-bovine synaptophysin (Cat. no. 902 314; Boehringer Mannheim GmbH, Mannheim; 1/25 dilution), which was used as an 'irrelevant antibody' negative control for the laminin monoclonal antibodies. As a range of antibodies to laminin chains was used, which produced different staining patterns in the ovary, they were also able to act as controls for each other. Normal rabbit serum (Cat. no. R9133; Sigma Chemical Co.) or normal mouse serum (Cat. no. M5905; Sigma Chemical Co.) also served as negative controls.

\section{Immunohistochemistry}

All sections were post-fixed by immersion in ethanol (if using polyclonal antibodies) or acetone (if using monoclonal antibodies) for 20-30 min, rinsed in hypertonic PBS (10 mmol sodium/potassium phosphate $1^{-1}$ with $0.274 \mathrm{~mol} \mathrm{NaCl} 1^{-1}$,

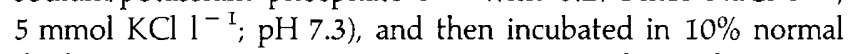
donkey serum (Cat. no. D9663; Sigma Chemical Co.) in hypertonic PBS $(30 \mathrm{~min})$. The sections were then incubated overnight with one of the primary antibodies (diluted in hypertonic PBS), rinsed in hypertonic PBS $(3 \times 5 \mathrm{~min})$, and then incubated as appropriate with either Fluorescein (DTAF)conjugated AffiniPure donkey anti-rabbit IgG (Cat. no. 711095-152; Jackson ImmunoResearch Laboratories, West Grove, PA; 1/150 dilution in hypertonic PBS; $45 \mathrm{~min}$ ) or with Cy3-conjugated AffiniPure donkey anti-mouse IgG (Cat. no. 715-165-150; Jackson ImmunoResearch; 1/150 dilution in hypertonic PBS; $45 \mathrm{~min}$ ). In some instances, sections that had been incubated with polyclonal antibodies were further incubated with biotin-SP-conjugated goat anti-rabbit $\operatorname{IgG}$ (Cat. no. 711-066-152; Jackson ImmunoResearch; 1/100 dilution in hypertonic PBS; $45 \mathrm{~min}$ ) to achieve further amplification of the immunostaining. Sections were then rinsed in hypertonic PBS $(3 \times 5 \mathrm{~min})$ and further incubated with $\mathrm{Cy} 3$ conjugatedstreptavidin (Cat. no. 016-160-084; Jackson ImmunoResearch; 1/100 dilution in hypertonic PBS; $1 \mathrm{~h}$ ). All incubations were at room temperature. All sections were finally rinsed in hypertonic PBS $(3 \times 5 \mathrm{~min})$, and mounted with buffered glycerol (0.167 mol Na $\mathrm{CO}_{3} \mathrm{l}^{-1}$ in $67 \%$ glycerol, $\mathrm{pH} 8.6$ ).

\section{Dual labelling}

As the $\alpha 1$ and $\beta 2$ staining of the preantral follicular basal lamina was very faint, and it was conceivable that not all follicles of this stage were labelled, dual labelling of the $\alpha 1$ chain with the $\gamma 1$ chain (which gave more intense staining of the basal laminae of preantral follicles; see results), and then dual labelling of the $\alpha 1$ and $\beta 2$ laminin chains was conducted. In addition, sections were dual labelled for the $\beta 2$ laminin chain and factor VIII-related antigen to confirm that the former was labelling the basal lamina of the vasculature. The protocol for dual labelling was essentially the same as described above, except that the sections were incubated concurrently with two primary antibodies, and that the sections were incubated concurrently with Fluorescein (DTAF)-conjugated AffiniPure donkey anti-rabbit IgG and Cy3-conjugated AffiniPure donkey anti-mouse IgG. Control sections were: the relevant polyclonal primary antibody and anti-rabbit secondary antibody; the relevant monoclonal primary antibody and anti-mouse secondary antibody; the polyclonal primary antibody and anti-mouse secondary antibody; the monoclonal primary antibody and anti-rabbit secondary antibody; the normal rabbit serum primary antibody and anti-rabbit secondary antibody; the normal mouse serum primary antibody and anti-mouse secondary antibody; both the normal rabbit serum and normal mouse serum primary antibodies; and both the anti-rabbit and antimouse secondary antibodies.

\section{Photography}

Sections were observed and photographed with an Olympus Vanox AHBT3 fluorescence microscope with Olympus C35AD-4 camera attachment, using the IB filter to excite the DTAF fluorochrome and the $G$ filter to excite the Cy3 fluorochrome. Photographs were taken using Kodak T-Max 400 black and white film.

\section{Electron microscopy}

Three bovine ovaries were processed for electron microscopy as reported by van Wezel and Rodgers (1996). Briefly, the ovarian artery was cannulated, and the associated ovary was flushed with Earle's balanced salt solution (EBSS) and then $2.5 \%(\mathrm{v} / \mathrm{v})$ glutaraldehyde in $0.1 \mathrm{~mol}$ 4-morpholinepropanesulfonic acid $\mathrm{l}^{-1}$. Tissue from the ovarian cortex was then cut into pieces approximately $1 \mathrm{~mm} \times 0.5 \mathrm{~mm} \times 2 \mathrm{~mm}$, and these were postfixed with osmium tetroxide, dehydrated and embedded in epoxy resin by standard methods as reported in detail by van Wezel and Rodgers (1996). Thick sections $(2 \mu \mathrm{m})$ were stained with $1 \%(\mathrm{w} / \mathrm{v})$ methylene blue, and thin sections were stained with uranyl acetate and lead nitrate and observed and photographed with a JOEL (Peabody, MA) CS1200 electron microscope.

\section{Results}

\section{General observations}

Five individual laminin chains were immunolocalized in bovine ovaries. In addition, an antibody raised against EHS laminin and, therefore, detecting the $\alpha 1, \beta 1$ or $\gamma 1$ chains, was used as a positive control. The results presented here are confined to follicles and the basal lamina structures therein, including the follicular basal lamina, basal laminae associated with endothelial cells and smooth muscle cells of the vasculature, and lamina-like material in the theca in areas where there are no recognized conventional basal laminae. Each of the primary antibodies used in this study resulted in staining of the follicular basal lamina which was abolished when that antibody was either omitted entirely from the immunostaining protocol or replaced by normal rabbit serum or normal mouse serum. The staining patterns produced by the different antibodies differed from each other when preantral follicles $(n>150$; Fig. 1), antral follicles that were 'healthy' ( $n=9$; Fig. 2 ) or 'atretic' $(n=10$; Fig. 3) were compared. Cells of the ovarian surface epithelium in normal rabbit serum controls were stained and, 

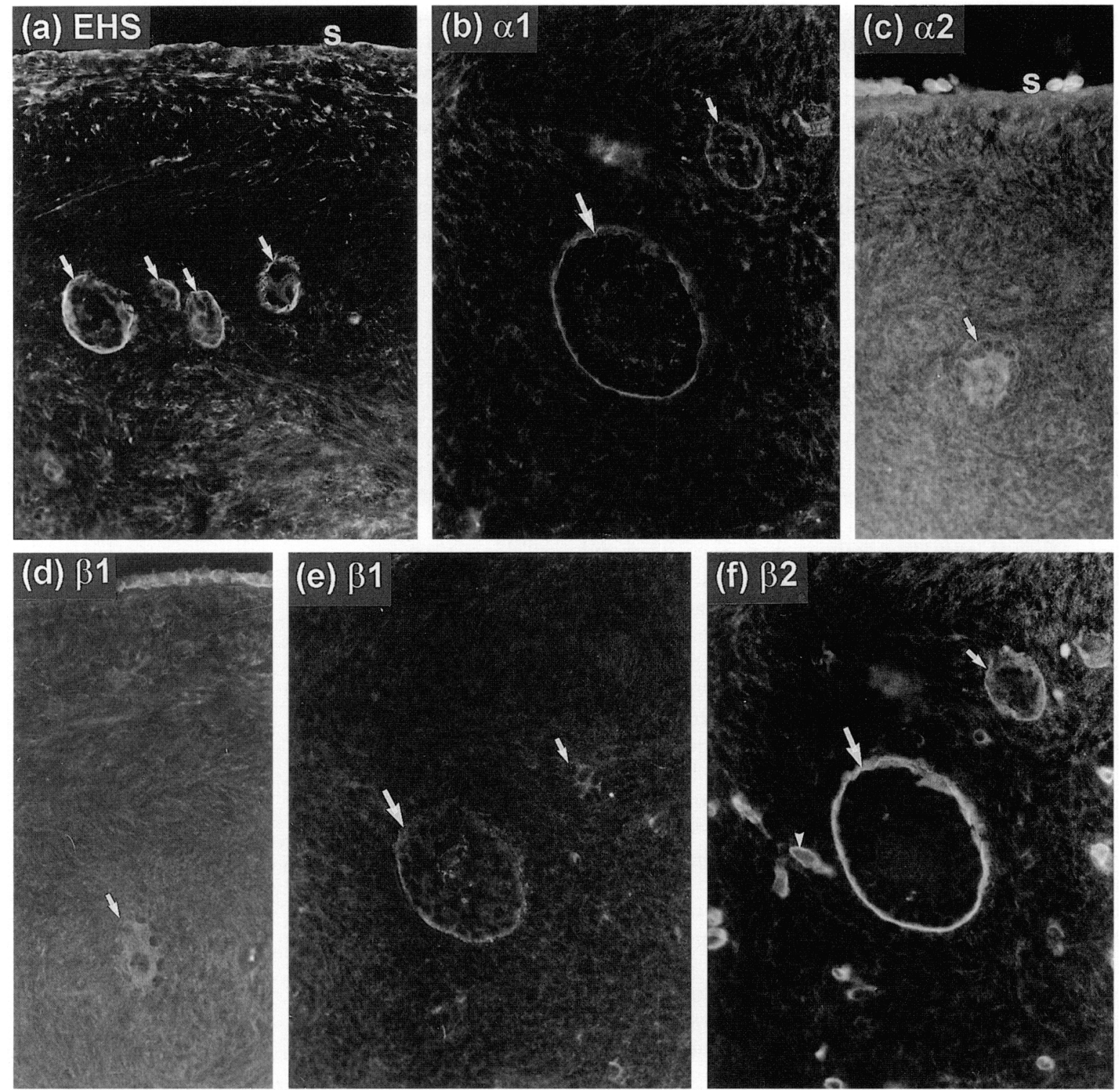

(e) $\beta 1$
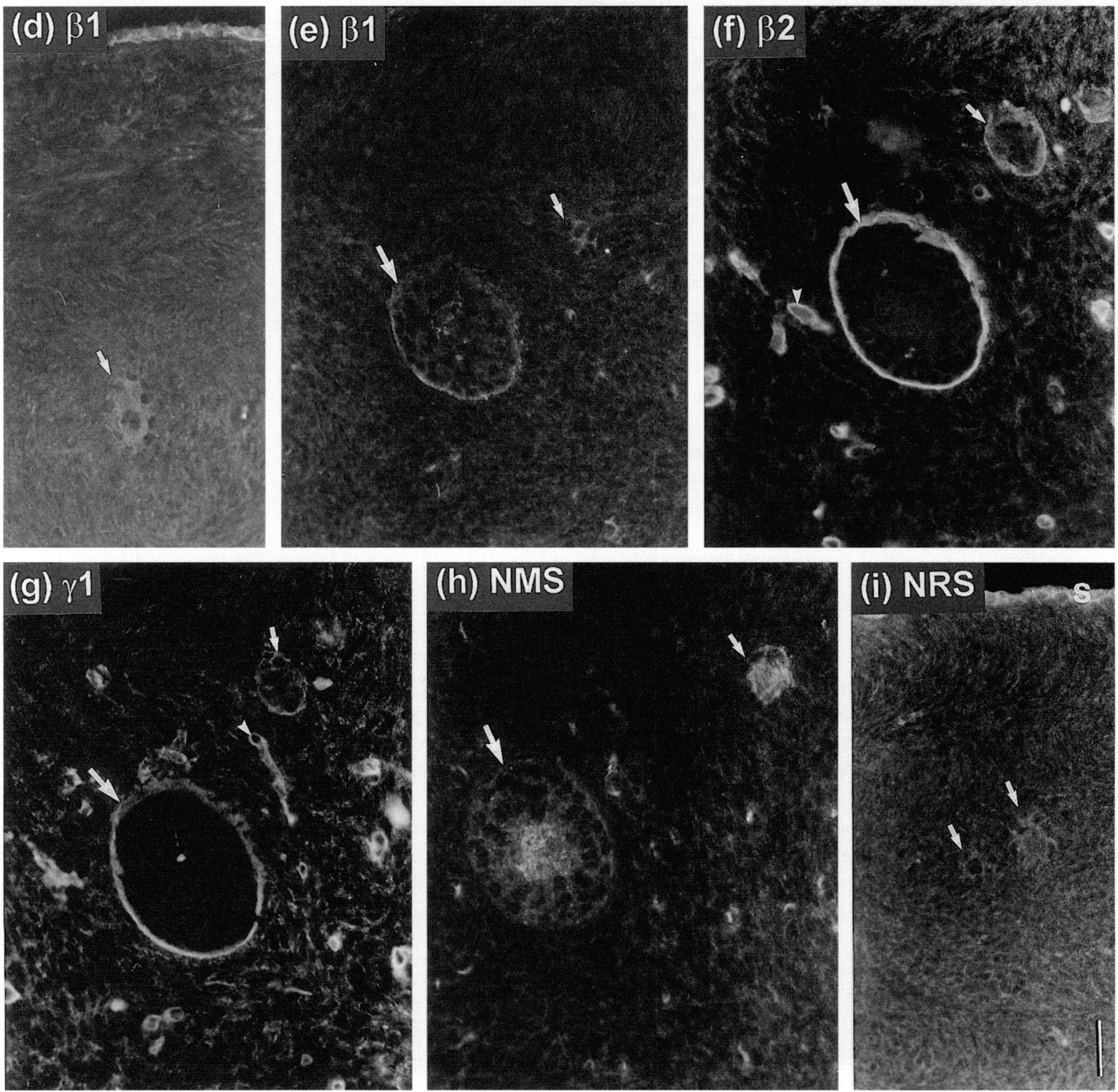

(i) NRS

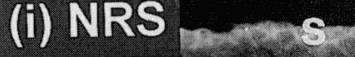


therefore, staining of the surface epithelium produced by using the $\alpha 1$ or $\alpha 2$ antibodies could not be considered as specific. The follicular staining patterns produced by each antibody are summarized (Table 1).

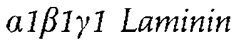

When the antibody directed against EHS laminin (laminin-I; Burgeson et al., 1994) was used, staining was present in the follicular basal lamina of all preantral follicles (Fig. 1a) and healthy antral follicles (Fig. 2a). The follicular basal lamina of atretic antral follicles also stained, but with great variation in intensity between follicles (Fig. $3 g, h$ ). The theca also stained positively using the EHS antibody, with staining in areas where there are no recognized conventional basal laminae (Fig. 3g,h).

\section{a1, a2 Laminin chains}

The $\alpha I$ (Fig. Ib) but not the $\alpha 2$ (Fig. Ic) laminin chain was immunolocalized to the basal lamina of preantral follicles. The $\alpha 1$ staining was usually not very intense, in contrast to strong staining for $\gamma 1$ in the primordial follicular basal lamina. Owing to the variation in intensity of staining of $\alpha 1$, the possibility that the $\alpha 1$ chain might not be present in some preantral follicles was considered. Sections were dual-stained for both the $\alpha I$ and $\gamma 1$ chains and this confirmed that the $\alpha 1$ chain was localized to all the preantral follicles that were positive for the $\gamma 1$ chain. The $\alpha I$ chain was also present in the follicular basal lamina of healthy antral follicles (Fig. 2b) and stained with a much greater intensity than at the preantral follicle stage. Of the nine healthy antral follicles examined, the basal laminae of only two were positive for the $\alpha 2$ laminin chain and, in these cases, the staining was not evenly distributed from one region to another; the basal laminae of the other healthy antral follicles were unstained (Fig. 2c). Both the $\alpha 1$ (Fig. 3b) and the $\alpha 2$ (Fig. 3c) chains were localized in the basal laminae of all of the ten atretic antral follicles examined. Punctate fluorescence was observed in the membrana granulosa of some atretic follicles (Fig. 3b), but this was not specific staining as it was also seen in control sections (Fig. 3a). The $\alpha I$ staining along the basal lamina was generally wider and less intense in atretic follicles than in healthy antral follicles.

\section{$\beta 1, \beta 2$ Laminin chains}

The $\beta 1$ chain of laminin was not detected in the follicular basal laminae of any of the small preantral follicles (Fig. Id,e), but large preantral follicles were stained weakly (Fig. 1e). In contrast, the $\beta 2$ chain was detected in the basal laminae of all preantral follicles that were positive for the $\alpha I$ laminin chain (and all $\alpha 1$-positive preantral follicles had been positive for $\gamma 1$ ) and the staining intensity increased with increasing size of the follicle (Fig. If). In both healthy and atretic antral follicles, the $\beta 1$ staining was present in the vasculature, which at times closely abutted the follicular basal lamina. However, staining of the follicular basal lamina itself was minimal or absent (Figs $2 \mathrm{~d}, \mathrm{e}$ and $3 \mathrm{~d}$ ). In contrast, the $\beta 2$ chain was detected in both the follicular basal lamina and the vasculature (Figs $2 \mathrm{f}, \mathrm{h}$ and $3 \mathrm{e}$ ). Vascular staining for the $\beta 1$ chain was limited to the endothelial cell basal laminae, while the $\beta 2$ chain was also present in the smooth muscle layers of the arterioles (compare Fig. 3d with 3e). Blood vessels throughout the theca and ovarian stroma were positive for factor VIII-related antigen, with all $\beta 2$ laminin localizing adjacent to cells positive for factor VIIIrelated antigen (Fig. $2 \mathrm{~h}, \mathrm{i}$ ). Similar to the pattern of $\alpha 1$ staining, the region of $\beta 2$ staining of the follicular basal lamina was wider and the staining was less intense in atretic follicles than in healthy follicles.

\section{$\gamma 1$ Laminin chain}

The follicular basal laminae of all the preantral follicles (Fig. Ig) and healthy antral follicles (Fig. 2g) observed stained strongly for the $\gamma 1$ laminin chain. In atretic antral follicles, staining of the follicular basal lamina was considerably less intense than in healthy antral follicles (Fig. 3f). Staining that was widely dispersed throughout the theca of healthy and atretic antral follicles, and not limited to the vasculature or other structures with distinct basal laminae, was also observed.

\section{Electron microscopy}

In general, the follicular basal lamina of primordial follicles was continuous and closely abutted the granulosa cells to envelop the follicle. The lamina appeared in some regions of most follicles to be composed of only a single layer of material while, in other regions, several layers of lamina material were observed. In regions where there were several layers of basal lamina material, these usually lay close together. Within growing follicles in most regions, the follicular basal lamina was generally multilayered. Often these layers were interconnected (Fig. 4). The inner layer of basal lamina closely abutted the granulosa cells, as for primordial follicles, but the other layers were not usually tightly packed and often this

Fig. 1. Immunofluorescent localization of laminin- 1 (EHS laminin, composed of $\alpha 1 \beta 1 \gamma 1$; rabbit polyclonal antisera), and individual laminin chains ( $\alpha 1$ and $\alpha 2$ are rabbit polyclonal antisera; $\beta 1, \beta 2$ and $\gamma 1$ are mouse monoclonal antisera) to preantral bovine follicles. (a) Positive staining of the follicular basal laminae for laminin-1 (EHS laminin) (b) Positive staining for the $\alpha 1$ chain but (c) no detectable $\alpha 2$ chain. Staining of the ovarian surface epithelial cells in (c) is also seen in the normal rabbit serum (NRS) control (i) and, therefore, could not be considered as specific. (d, e) Nonspecific staining between the granulosa cells in small preantral follicles, which is also seen in the normal mouse serum control (h), but no staining of the basal lamina for the $\beta I$ chain. In contrast, the larger preantral follicle shown in (e) was positive for this chain, but the slight staining of the oocyte was also seen in the normal mouse serum (NMS) control (h), and considered nonspecific. ( $f, g$ ) Positive staining of the follicular basal lamina for the $\beta 2$ and $\gamma 1$ chains, respectively, and staining of the stromal vasculature. The follicles in (c, d, i) were exposed to show the outline of the follicles and do not reflect high background concentrations. Arrows indicate the position of the follicular basal laminae, small arrows to small preantral follicles, large arrows to large preantral follicles; arrowheads indicate the stromal vasculature; $\mathrm{S}=$ the ovarian surface epithelium. Scale bar represents $50 \mu \mathrm{m}$. 


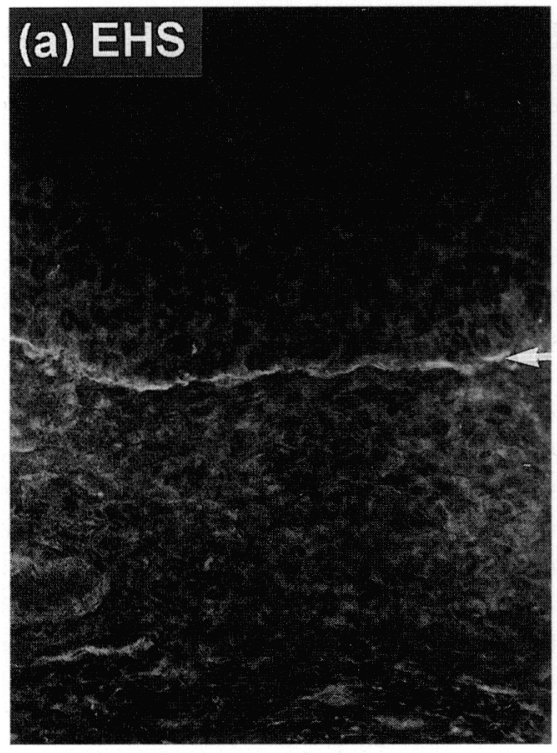

(b) $\alpha 1$

(c) $\alpha 2$
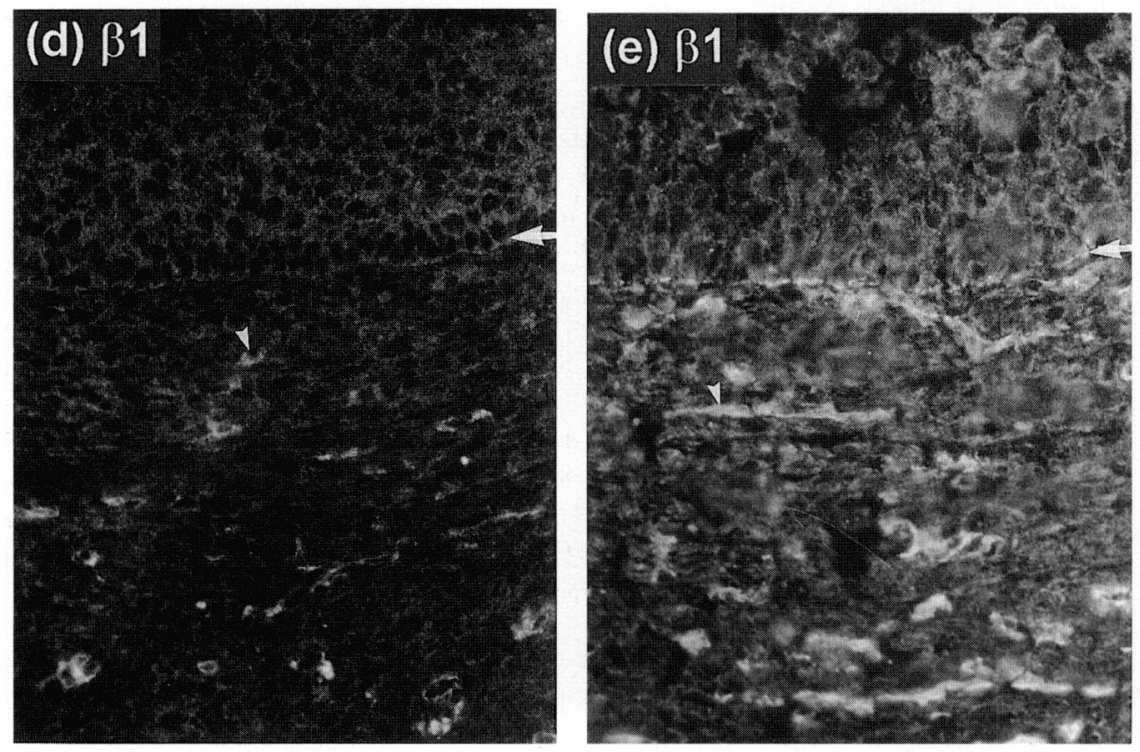

(f) $\beta 2$
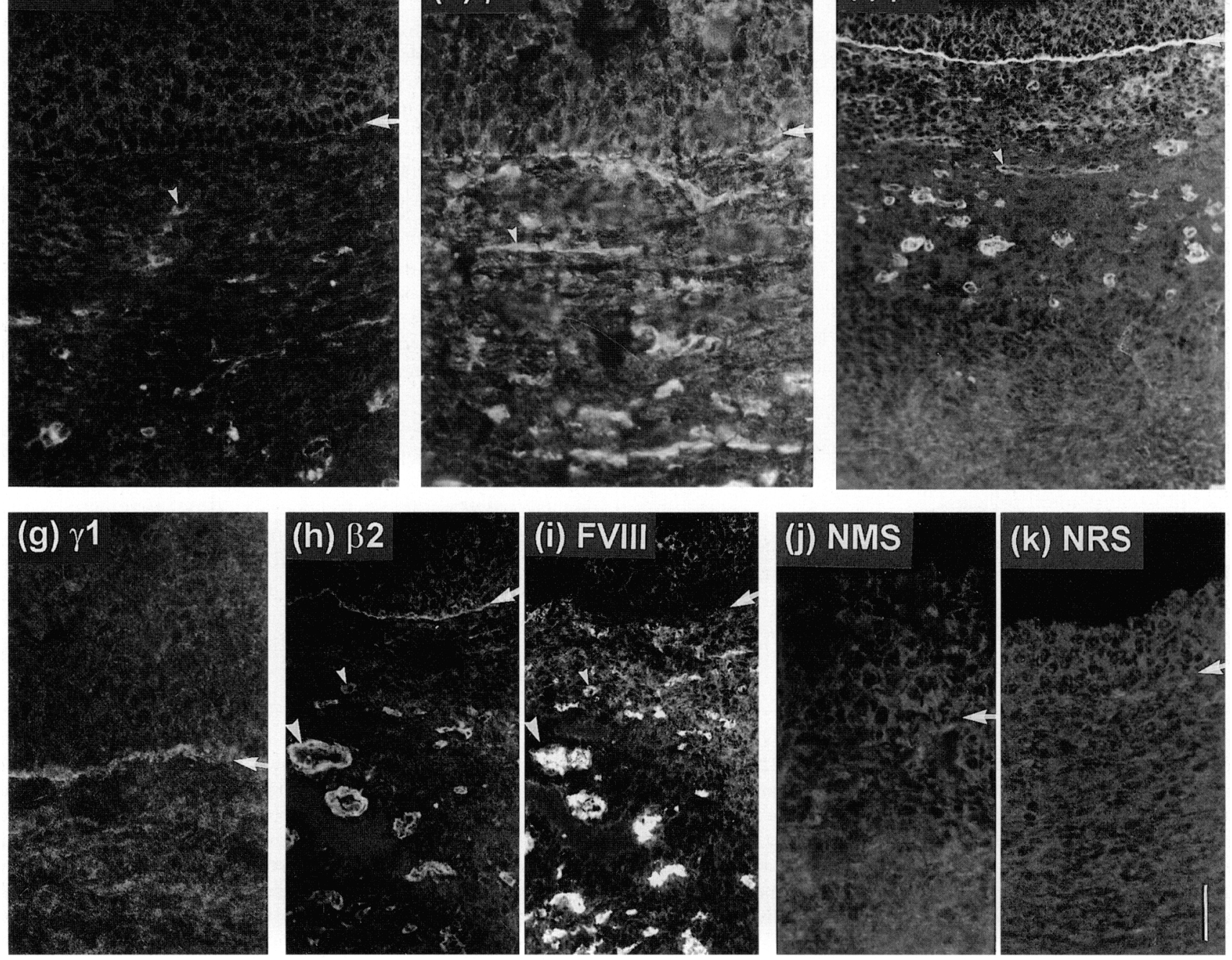

(h) $\beta 2$ 
region of basal lamina layers spanned $1-2 \mu \mathrm{m}$ in cross-section. The arrangement of the basal lamina layers differed among follicles: some follicles apparently having sheets of basal lamina that ran parallel to the basal surface of the membrana granulosa, while the basal lamina layers of other follicles were more convoluted (Fig. 4).

\section{Discussion}

This is the first study to immunolocalize a range of individual laminin chains to the follicular basal lamina, and to investigate the changing composition of this basal lamina during follicular development and atresia. The $\alpha 1$ chain was present in the follicular basal lamina at all stages of follicle development, while the $\alpha 2$ chain was present only in atretic antral follicles and a few healthy antral follicles and absent from primordial and growing preantral follicles. The $\beta 2$ chain was present in the follicular basal lamina of follicles of all stages, but the $\beta I$ chain was detected only in the basal lamina of large preantral follicles. Staining for the $\alpha 1, \beta 2$ or $\gamma 1$ chains appeared to increase in intensity from the preantral to healthy antral stages, and the basal lamina of atretic antral follicles appeared thicker but stained less intensely than that of healthy follicles. The basal laminae of the thecal vasculature stained positively for the $\beta 1$ and $\beta 2$ chains, while the $\gamma 1$ chain localized more generally throughout the theca in areas that do not have a recognized conventional basal lamina.

Fröjdman et al. (1995) showed that mouse primordial, primary and antral follicles contain the $\alpha 1$ chain and, in the present study, we have shown a similar result in the bovine ovary. Antral follicles contain several layers of granulosa cells, and those cells adjacent to the follicular basal lamina differ from those adjacent to the follicular antrum in shape (columnar versus rounded or flattened; M. Krupa, I. L. van Wezel and R. J. Rodgers, unpublished) and the expression of a range of genes (Amsterdam et al., 1975; Bortolussi et al., 1977; Zoller and Weisz, 1978, 1979; Zlotkin et al., 1986). Studies using kidney cells suggest that the $\alpha 1$ laminin chain may have a role in maintaining this polarity of the granulosa layer. Kidney cells in culture became polarized when laminin $\alpha 1$ was expressed, and antibodies to laminin $\alpha 1$ prevented polarization (Klein et al, 1988, 1990; Ekblom, 1989).

Laminin $\alpha 2$ (merosin in the old nomenclature; Burgeson et al., 1994) was found in the follicular basal lamina of atretic follicles and in a few healthy antral follicles. These follicles also had the laminin $\alpha I$ chain; at this point it is unclear whether both chains were being produced in these follicles or whether, before the onset of atresia, there was a switch from the production of $\alpha I$ to $\alpha 2$ chains, and no ongoing production of the $\alpha 1$ chain. It is not clear whether laminin $\alpha 2$ plays a major role in the induction of follicular atresia, or is expressed as a consequence of atresia. An alternative explanation is that the laminin $\alpha 2$ chain is produced as a normal event in late follicular development, and its expression coincides with atresia only because atresia of these follicles is commonplace. Evidence from other tissues suggests that this could be the case, as the $\alpha 2$ chain plays a role when cells are differentiating or maturing (Ehrig et al., 1990). Thus, there is no expression of $\alpha 2$ by cultured cell lines, limited expression in malignant tumours, and expression in mouse basal laminae occurs postnatally not prenatally (Leivo and Engvall, 1988; Leivo et al., 1989a,b). Similarly, in the epithelium of the human small intestine, whereas expression of the $\alpha 1$ chain is evident at 7 weeks of gestation, the $\alpha 2$ chain is not present until 16 weeks of gestation (Perreault et al., 1995). However, to ascertain the role of the laminin $\alpha 2$ chain in the follicular basal lamina, it will be necessary to study follicles of known developmental stage.

The observation that both $\beta 1$ and $\beta 2$ laminin chains were present in the follicular basal lamina before antrum formation, but only the $\beta 2$ chain was present after antrum formation, parallels previous studies in the developing kidney: that is, the laminin $\beta I$ chain was expressed at three early stages (the comma and s-shaped structures, and the capillary loop) and $\beta 2$ was expressed later as the glomerulus developed (see Noakes et al., 1995). Furthermore, Noakes et al. (1995) showed that changes in $\beta 1$ and $\beta 2$ expression are important for proper filtering of plasma proteins by the glomerular basement membrane. Others have shown that cultured mouse embryonic lung cells can be induced to polarize and form a lumen by treatment with a fragment of laminin $\beta 1$ and heparan sulfate proteoglycan (Schuger et al., 1996). On the basis of these studies, it is conceivable that changes in laminin $\beta 1$ or $\beta 2$ concentration during follicular development are important in controlling the filtration properties of the follicular basal lamina and initiating the formation of the follicular antrum.

The present study localizing individual laminin chains did not identify which laminin type was present in the follicular basal lamina. Laminin-3 (s-laminin; $\alpha 1 \beta 2 \gamma 1$; Burgeson et al., 1994) may be present in the follicular basal lamina, as suggested by the presence of $\alpha 1, \beta 2$ and $\gamma 1$ chains throughout follicular development. However, the presence of the $\alpha 2$ and $\beta 1$ chains in the follicular basal lamina at specific stages of development indicated that other laminin isoforms were present, at least during select stages. The $\alpha 3, \alpha 4, \alpha 5, \beta 3$ and $\gamma 2$ laminin chains have not yet been examined in the follicular

Fig. 2. Immunofluorescent localization of laminin- 1 and individual laminin chains to healthy bovine antral follicles. In each photograph, the location of the basal lamina is indicated by the arrow on the right side. The membrana granulosa lies above the basal lamina and the theca below it, and the follicular antrum is uppermost. (a) Positive staining of the follicular basal lamina for laminin-1 (EHS laminin). (b) Positive staining in the follicular basal lamina for the $\alpha 1$ chain but (c) no detectable $\alpha 2$ chain. (d,e) The $\beta 1$ laminin chain is undetectable in the follicular basal lamina of these follicles, although the thecal vasculature is stained positively and in some places this staining is very closely apposed to the follicular basal lamina. ( $f, h$ ) Positive staining for the $\beta 2$ chain in the follicular basal lamina, and the vasculature (including arteriole smooth muscle basal lamina) as determined by dual labelling for the endothelial cell marker factor VIII-related antigen in (i), the same section as (h). ( $\mathrm{g}$ ) The $\gamma 1$ antibody was also immunolocalized to the follicular basal lamina. Controls: (j) normal mouse serum (NMS) and (k) normal rabbit serum (NRS). Small arrowheads indicate the vasculature, large arrowheads indicate the arterioles with smooth muscle. Scale bar represents $50 \mu \mathrm{m}(\mathrm{a}-\mathrm{e}, \mathrm{g}$, j, $\mathrm{k}$ ) and $100 \mu \mathrm{m}$ (f, h, i). 

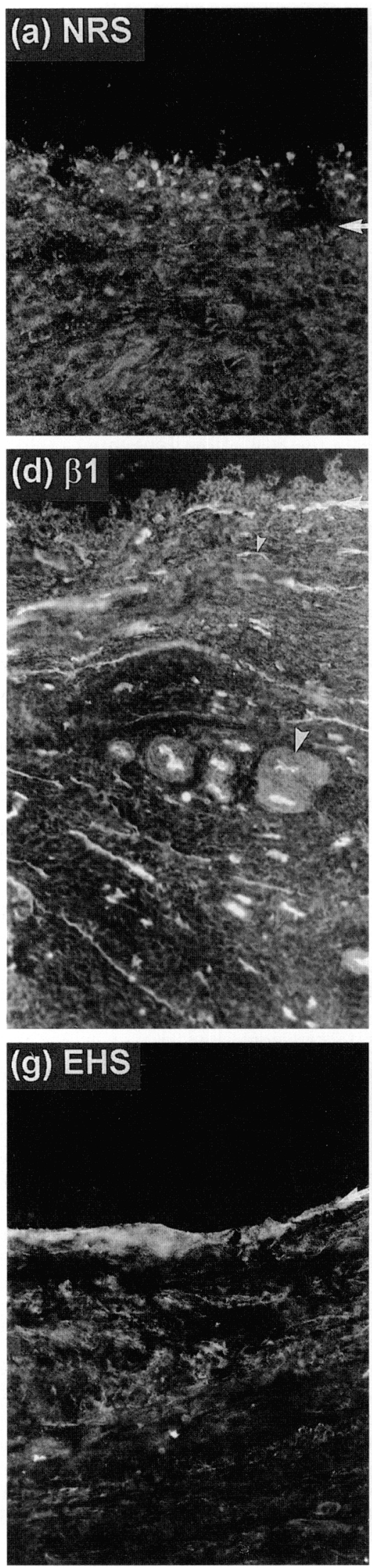

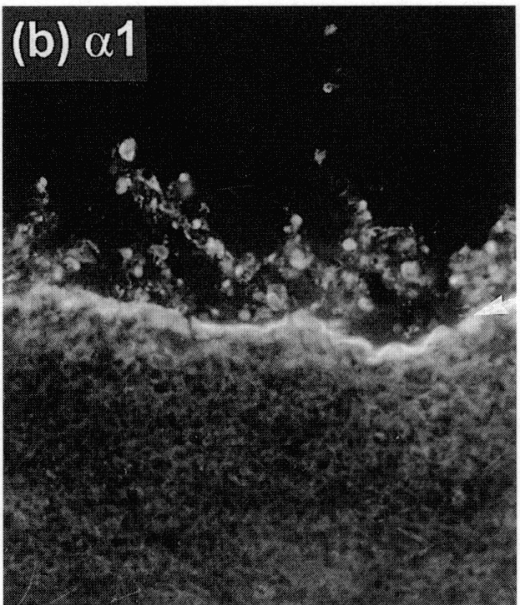

\section{(e) $\beta 2$}

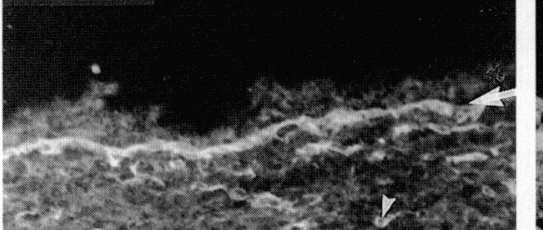

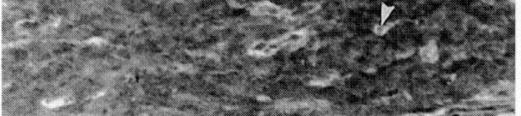

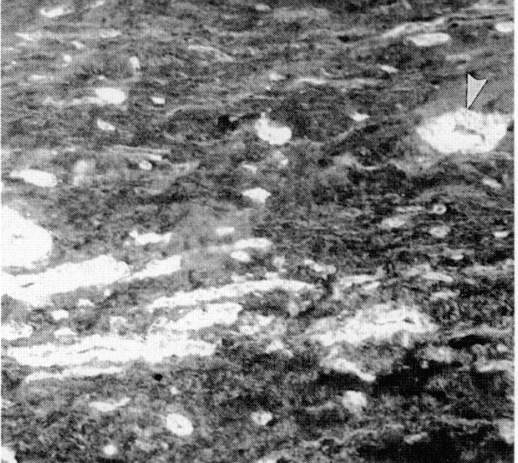
(h) EHS

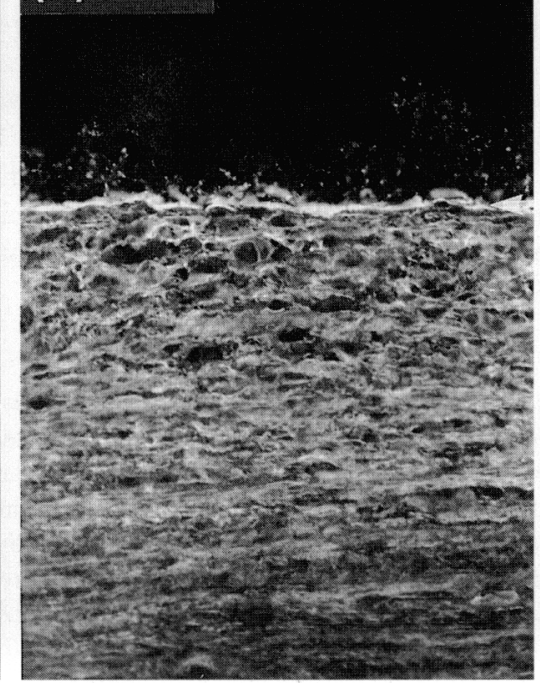

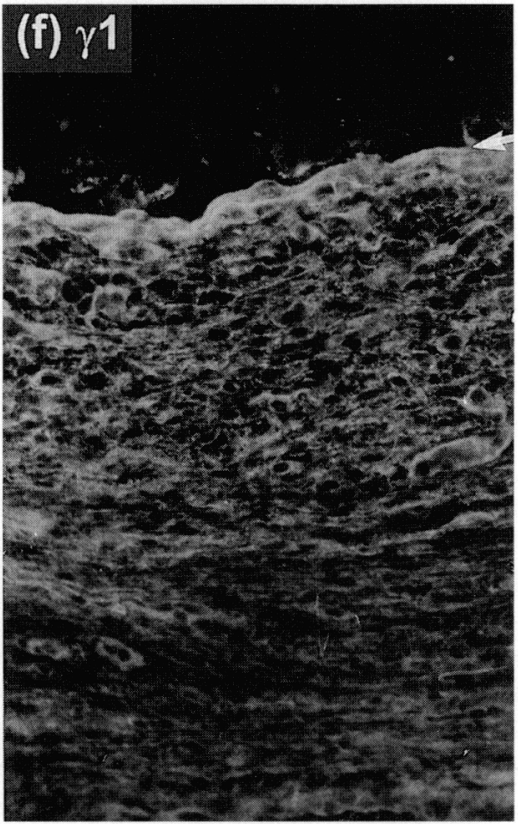

(i) NMS

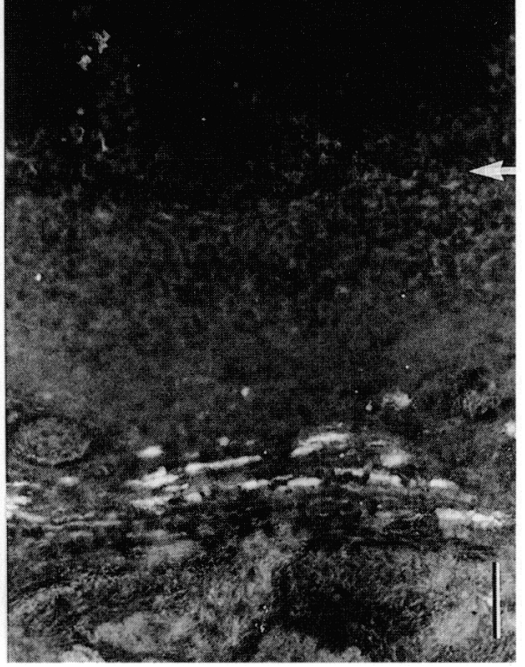


Table 1. Staining patterns produced by antisera specific to the individual $\alpha 1, \alpha 2, \beta 1, \beta 2$ and $\gamma 1$ laminin chains and to EHS ( $\alpha 1 \beta 1 \gamma 1)$ laminin in bovine ovarian follicles

\begin{tabular}{|c|c|c|c|c|c|c|}
\hline & $\alpha 1$ & $\alpha 2$ & $\beta 1$ & $\beta 2$ & $\gamma I$ & EHS \\
\hline \multicolumn{7}{|l|}{ Follicular basal lamina ${ }^{a}$} \\
\hline Primordial/primary & + & - & - & + & + & + \\
\hline Large preantral & ++ & - & + & ++ & ++ & ++ \\
\hline Healthy antral & +++ & $-1+$ & - & +++ & ++ & +++ \\
\hline Atretic antral & ++ & + & - & ++ & + & ++ \\
\hline \multicolumn{7}{|l|}{ Other follicular } \\
\hline Thecal matrix & - & - & - & - & ++ & ++ \\
\hline Vascular subendothelial & - & - & +++ & +++ & & \\
\hline Vascular smooth muscle & - & - & - & +++ & & \\
\hline
\end{tabular}

Intensity of staining: - , none; + , weak; ++ , moderately intense; +++ , very intense. Comparison of intensity should only be made within a column (that is, between follicles of different classes) and not within a row (that is, between laminin chains) as different antisera have been used.

${ }^{a}$ The same nine healthy and ten atretic antral follicles were examined for each anitbody. $>150$ preantral follicles were examined for each antibody (these were not necessarily the same follicles).

basal lamina of any species, but may also contribute to laminin molecules in the follicular basal lamina. Gene expression for the laminin $\alpha 4$ chain has been detected in the ovary (Iivanainen et al., 1995). However, whether this is the follicular basal lamina or one of the many other types of basal lamina or non-lamina structures in the ovary is not known.

Evidence presented here and previously (van Wezel and Rodgers, 1996) suggests that the basal lamina is continually remodelled during follicular development. Electron microscopic examination of the bovine follicular basal lamina has found that it is closely associated with the granulosa cells in primordial follicles (van Wezel and Rodgers, 1996). The follicular basal lamina of larger follicles was composed of many layers of basal lamina material joined together to form a branching network (this study and see Rodgers et al., 1995), which is consistent with the notion that the basal lamina is shed and replaced by a newly synthesized basal lamina closer to the granulosa cells. Under the light microscope, multilayers of basal lamina would only have been detected as either an increased thickness or increased intensity of staining, as observed for the laminin $\alpha I$, $\beta 2$ and $\gamma 1$ chains during follicular development. However, the more obvious explanation that this staining pattern was due to increased amounts of the specific laminin chains per unit surface area of basal lamina requires further investigation. Increasing thickness of the basal lamina staining during follicular atresia was also observed. This finding, together with the decreased intensity of staining for the $\alpha 1, \beta 2$ and $\gamma 1$ in atretic follicles, suggests that there may have been degradation of some basal lamina components - perhaps the collagen IV backbone causing the basal lamina to lose its compact structure. Alternatively, simple convolution of the basal lamina may have occurred when the follicular fluid was resorbed and the atretic follicle decreased in size, producing the apparent increase in thickness of the basal lamina.

The cellular origin of the follicular basal lamina is a contentious issue (van Wezel and Rodgers, 1996). Within the laminin component of the follicular basal lamina, northern blot analysis detected the expression of the $\gamma 1$ (old nomenclature: B2) chain but not $\beta 1$ (old nomenclature: B1) by granulosa cells (Zhao and Luck, 1995), which is consistent with the present study localizing the $\gamma 1$ but not the $\beta 1$ laminin chain to the follicular basal lamina of antral follicles. Furthermore, a previous immunoelectron study in the rat ovary (Leardkamolkarn and Abrahamson, 1992) localized laminin to Call-Exner bodies, which are ultrastructurally similar to basal lamina and have been observed within the membrana granulosa of antral follicles in vivo in cows (I. L. van Wezel and R. J. Rodgers, unpublished) and rabbits (Gosden et al., 1988). Laminin was also localized intracellularly in both granulosa and thecal cells, but this latter observation may represent degradation rather than synthesis. The contribution of the theca, or indeed the stroma surrounding preantral follicles, to the production of the follicular basal lamina is not known. In other systems, it is predominantly epithelial rather than stromal cells that

Fig. 3. Immunofluorescent localization of individual laminin chains and laminin-1 (EHS laminin) to atretic bovine antral follicles. In each photograph, the basal lamina is indicated by the arrow on the right side; the membrana granulosa lies above the basal lamina and the theca below it, and the follicular antrum is uppermost. (a) Normal rabbit serum (NRS) control showing punctate staining in the membrana granulosa. Similar staining for any laminin chain was, therefore, not considered as specific. Positive staining of the basal lamina for the $\alpha 1$ (b) and $\alpha 2$ chain (c). (d) No continuous staining of the basal lamina for the $\beta 1$ laminin chain but localization of this chain to the vasculature, some of which closely abuts the follicular basal lamina. The smooth muscle of arterioles is not stained. (e) $\beta 2$ chain localized to the follicular basal lamina and the vasculature, including arteriole smooth muscle. (f) Weak staining pattern produced by antibodies directed against $\gamma 1$ laminin. $(g, h)$ Localization patterns produced by antibodies directed against laminin-1, with staining of the basal lamina being either very weak ( $\mathrm{g}$ ) or very strong $(\mathrm{h}$ ) relative to the thecal staining. (i) Normal mouse serum (NMS) control. Small arrowheads indicate the vasculature, large arrowheads indicate the vasculature with smooth muscle. Scale bar represents $50 \mu \mathrm{m}(\mathrm{a}-\mathrm{c}, \mathrm{f}-\mathrm{h})$ and $100 \mu \mathrm{m}(\mathrm{d}, \mathrm{e}, \mathrm{i})$. 


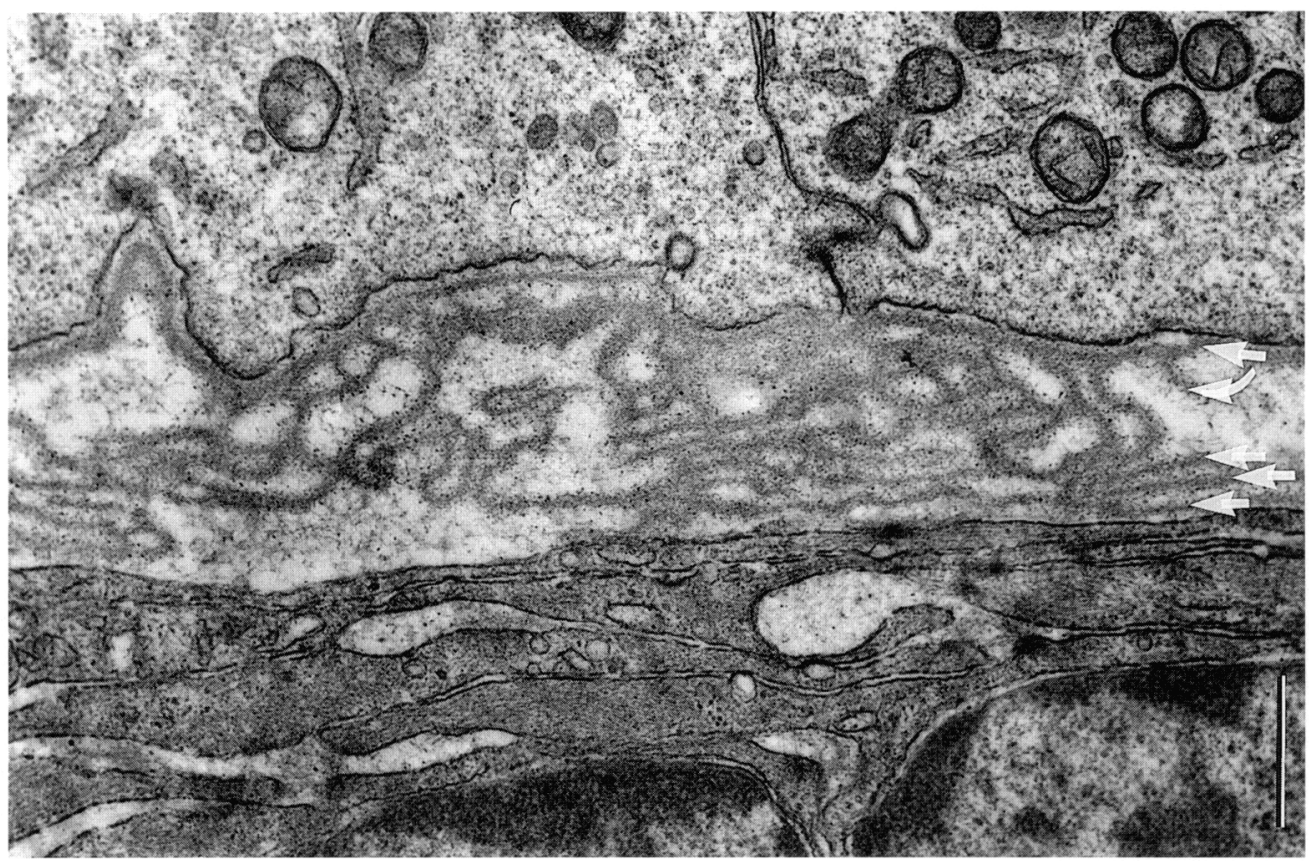

Fig. 4. Electron micrograph of the follicular basal lamina of a growing bovine preantral follicle. The many layers of basal lamina are indicated by arrows. Granulosa cells are uppermost, and thecal-stromal cells are beneath the follicular basal lamina. Scale bar represents $0.5 \mu \mathrm{m}$.

synthesize basal lamina components, although in some tissues both cell types make a contribution (Timpl and Dziadek, 1986). The thecal compartment of antral follicles has been shown by northern analyses to express laminin $\beta 1$ and $\gamma 1$ chains (Zhao and Luck, 1995). The present study showed laminin $\beta 1$ in the thecal vasculature but not in the follicular basal lamina of antral follicles, suggesting that the thecal expression of $\beta 1$ is not necessarily a contribution to the follicular basal lamina. Similarly, laminin $\gamma 1$ was found widely distributed in the theca and, although it is present in the follicular basal lamina, expression in the theca cannot be considered as proof that the theca contributes laminin $\gamma I$ to the follicular basal lamina. However, it is possible that the follicular basal lamina in vivo requires a contribution from both the granulosa cells and the stroma or thecal cells.

Laminin chains were also detected in the theca interna and externa of antral follicles. Both $\beta 1$ and $\beta 2$ were localized to subendothelial basal laminae of the blood vessels (arterioles, capillaries and venules). The laminin $\beta 2$ chain was also localized to the smooth muscle layers surrounding arterioles, which were located principally in the theca externa, consistent with studies in other tissues, in which the $\beta 1$ chain was limited to subendothelial basal laminae, while the $\beta 2$ chain was present between smooth muscle cells (Walker-Caprioglio et al., 1995). Staining for the laminin $\gamma 1$ chain or EHS laminin was present throughout the theca interna and externa in areas where there are no recognized conventional basal laminae. This pattern of staining has been observed using antibodies to some types of collagen IV (H. F. Rodgers and R. J. Rodgers, unpublished) and to EHS $(\alpha 1 \beta 1 \gamma 1)$ laminin in rats (Bagavandoss et al., 1983; Leardkamolkarn and Abrahamson, 1992) and mice (Fröjdman et al., 1995), and in the interstitial tissue of developing gonads in these species (Fröjdman et al., 1989, 1992a,b, 1993, 1995;
Smith and MacKay, 1991). It is possible that this staining in the theca is due to free $\gamma 1$ chains or free laminin not associated with a basal lamina. However, it is also possible that it is associated with small fragments of basal lamina-like, electron-dense material found extracellularly in the theca. This material has been observed by electron microscopy in the theca of sheep (O'Shea et al., 1978), rats (Leardkamolkarn and Abrahamson, 1992) and cows (see Fig. 1 in Rodgers et al., 1986). The origins and functions of this 'thecal matrix' are not known.

In conclusion, this is the first detailed immunolocalization study of individual members of any of the broad classes of basal lamina components, in the ovarian follicle. The observations made here confirm and extend previous studies, showing that the $\alpha 1, \alpha 2, \beta 1, \beta 2$ and $\gamma 1$ laminin chains are differentially expressed in the ovarian follicle, and that the composition of the follicular basal lamina, at least in terms of laminin, changes during follicular development and atresia. On the basis of studies using other cell types it seems likely that the different laminin chains have specific roles in the regulation of antrum formation and the development of the follicular epithelium.

The authors thank the following people for donating antibodies used in this study: L. Sorokin ( $\alpha 1)$, M. Paulsson ( $\alpha 2)$, P. Davies $(\beta 1, \gamma 1)$ and P. Noakes $(\beta 2)$. This work was supported by grants from the Flinders Medical Centre Research Foundation and the National Health and Medical Research Council of Australia.

\section{References}

Amsterdam A, Koch Y, Lieberman ME and Lindner HR (1975) Distribution of binding sites for human chorionic gonadotropin in the preovulatory follicle of the rat Journil of Cell Biology $67894-900$ 
Amsterdam A, Rotmensch S, Furman A, Venter EA and Vlodavsky I (1989) Synergistic effect of human chorionic gonadotropin and extracellular matrix on in vitro differentiation of human granulosa cells: progesterone production and gap junction formation Endocrinology 124 1956-1964

Andersen MM, Krall J, Byskov AG and Faber M (1976) Protein composition in the fluid of individual bovine follicles Journal of Reproduction and Fertility $\mathbf{4 8}$ $109-118$

Bagavandoss P, Midgley AR, Jr and Wicha M (1983) Developmental changes in the ovarian follicular basal lamina detected by immunofluorescence and electron microscopy Journal of Histochemistry and Cytochemistry 31 633-640

Bortolussi M, Marini G and Dal Lago A (1977) Autoradiographic study of the distribution of LH (hCG) receptors in the ovary of untreated and gonadotropin-primed immature rats Cell and Tissue Research 183 329-342

Burgeson RE, Chiquet M, Deutzmann R, Ekblom P, Engel J, Kleinman H, Martin GR, Meneguzzi G, Paulsson M, Sanes J, Timpl R, Tryggvason K, Yamada $Y$ and Yurchenco PD (1994) A new nomenclature for the laminins Matrix Biology 14 209-211

Christiane Y, Demoulin A, Gillain D and Leroy F (1988) Laminin and type III procollagen peptide in human preovulatory follicular fluid Fertility and Sterility 50 48-51

Durbeej M, Fecker L, Hjalt T, Zhang H-Y, Salmivirta K, Klein G, Timpl R, Sorokin L., Ebendal T, Ekblom P and Ekblom M (1997) Expression of laminin $\alpha 1, \alpha 5$ and $\beta 2$ chains during embryogenesis of the kidney and vasculature Matrix Biology 15 397-413

Ehrig K, Leivo I, Argraves WS, Ruoslahti E and Engvall E (1990) Merosin, a tissue-specific basement membrane protein, is a laminin-like protein Proceedings of the National Academy of Science USA 87 3264-3268

Ekblom P (1989) Developmentally regulated conversion of mesenchyme to epithelium FASEB Journal 3 2141-2150

Engvall E (1993) Laminin variants: why, where and when? Kidney International $432-6$

Faddy MJ and Gosden RG (1995) A mathematical model of follicle dynamics in the human ovary Human Reproduction 10 770-775

Figueiredo JR, Hulshof SCJ. Thiry M, Van Den Hurk R, Bevers MM, Nusgens B, Beckers JF (1995) Extracellular matrix proteins and basement membrane: their identification in bovine ovaries and significance for the attachment of cultured preantral follicles Theriogenology 43 845-858

Fröjdman K, Paranko J, Kuopio T and Pelliniemi LJ (1989) Structural proteins in sexual differentiation of embryonic gonads International Journal of Developmental Biology 33 99-103

Fröjdman K, Malmi R and Pelliniemi LJ (1992a) Lectin-binding carbohydrates in sexual differentiation of rat male and female gonads Histochemistry 97 $469-477$

Fröjdman K, Paranko J, Virtanen I and Pelliniemi LJ (1992b) Intermediate filaments and epithelial differentiation of male rat embryonic gonad Differentiation 50 113-123

Fröjdman K, Paranko J, Virtanen I and Pelliniemi LJ (1993) Intermediate filament proteins and epithelial differentiation in the embryonic ovary of the rat Differentiation 55 47-55

Fröjdman K, Ekblom P, Sorokin L, Yagi A and Pelliniemi J (1995) Differential distribution of laminin chains in the development and sex differentiation of mouse internal genitalia International Journal of Developmental Biology 39 335-344

Gosden RG, Hunter RHF, Telfer E, Torrance C and Brown N (1988) Physiological factors underlying the formation of ovarian follicular fluid Journal of Reproduction and Fertility 82 813-825

Greenwald GS and Terranova PF (1988) Follicular selection and its control. In The Physiology of Reproduction pp 387-445 Eds E Knobil, JD Neill, Ewing LL, Greewald GS, Markert CL and DW Pfaff. Raven Press, New York

Hay ED (1991) Cell Biology of Extracellular Matrix 2nd edn. Plenum Press, New York

Hunter DD, Shah V, Merlie JP and Sanes JR (1989) A laminin-like adhesive protein concentrated in the synaptic cleft of the neuromuscular junction Nature 338 229-234

Iivanainen A, Sainio K, Sariola H, Tryggvason K (1995) Primary structure and expression of a novel human laminin alpha-4 chain FEBS Letters 365 183-188

Kaneko Y, Hirakawa S, Momose K and Konomi H (1984) Immunochemical localization of Type I, III, IV and V collagens in the normal and polycystic ovarian capsules Acta Obstetrica et Gynaecologica Japonica 36 2473-2474 (Abstract)

Klein G, Langegger M, Timpl R and Ekblom P (1988) Role of laminin A chain in the development of epithelial cell polarity Cell 55 331-341
Klein G, Ekblom M, Fecker I, Timpl R, and Ekblom P (1990) Differential expression of laminin $\mathrm{A}$ and $\mathrm{B}$ chains during development of embryonic mouse organs. Development $110823-837$

Leardkamolkarn V and Abrahamson DR (1992) Immunoelectron microscopic localization of laminin in rat ovarian follicles Anatomical Record $23341-52$

Leivo I and Engvall E (1988) Merosin, a protein specific for basement membranes of Schwann cells, striated muscle, and trophoblast, is expressed late in nerve and muscle development Proceedings of the National Academy of Science USA 85 1544-1548

Leivo I, Laurila P, Wahlström T and Engvall E (1989a) Expression of merosin, a tissue-specific basement membrane protein, in the intermediate trophoblast cells if choriocarcinoma and placenta Laboratory Investigation 60 426-432

Leivo I, Engvall E, Laurila P and Miettinen M (1989b) Distribution of merosin, a laminin-related tissue-specific basement membrane protein, in human Schwann cell neoplasms Laboratory Investigation 61 426-432

Leu FJ, Engvall E and Damjanov I (1986) Heterogeneity of basement membranes of the genitourinary tract revealed by sequential immunofluorescence staining with monoclonal antibodies to laminin Journal of Histochemistry and Cytochemistry 34 483-489

Lindblom A, Marsh T, Fauser C, Engel J, Paulsson M (1994) Characterization of native laminin from bovine kidney and comparison with other laminin variants European Journal of Biochemistry 219 383-392

Luck MR (1994) The gonadal extracellular matrix Oxford Reviews in Reproductive Biology 16 33-85

Noakes PG, Gautam M, Mudd J, Sanes JR and Merlie JP (1995) The renal glomerulus of mice lacking s-laminin/laminin $\beta 2$ : nephrosis despite molecular compensation by laminin $\beta 1$ Nature Genetics 10 400-406

O'Shea JD, Cran DG, Hay MF and Moor RM (1978) Ultrastructure of the theca interna of ovarian follicles in sheep Cell and Tissue Research 187 473-478

Palotie A, Peltonen L, Foidart J-M and Rajaniemi H (1984) Immunohistochemical localization of basement membrane components and interstitial collagen types in preovulatory rat ovarian follicles Collagen Related Research $\mathbf{4}$ 279-287

Paulsson M (1992) Basement membrane proteins: structure, assembly, and cellular interactions Critical Reviews in Biochemistry and Molecular Biology 27 93-127

Paulsson M and Saladin K (1989) Mouse heart laminin. Purification of the native protein and structural comparison with Engelbreth-Holm-Swarm tumor laminin Journal of Biological Chemistry 264 18 726-18 732

Perreault N, Vachon PH ansd Beaulieu JF (1995) Appearance and distribution of laminin A chain isoforms and integrin alpha 2, alpha 3, alpha 6, beta 1 , and beta 4 subunits in the developing human small intestinal mucosa Anatomical Record 242 242-250

Rajakoski E (1960) The ovarian follicular system in sexually mature heifers with special reference to seasonal, cyclical and left-right variations Acta Endocrinologica Supplementum 52 6-68

Richardson MC, Davies DW, Watson RH, Dunsford ML, Inman CB and Masson GM (1992) Cultured human granulosa cells as a model for corpus luteum function: relative roles of gonadotrophin and low density lipoprotein studied under defined culture conditions Human Reproduction 7 12-18

Rodgers HF, Lavranos TC, Vella CA and Rodgers RJ (1995) Basal lamina and other extracellular matrix produced by bovine granulosa cells in anchorageindependent culture Cell and Tissue Research 282 463-471

Rodgers RJ, Rodgers HF, Hall PF, Waterman MR and Simpson ER (1986) Immunolocalization of cholesterol side-chain-cleavage cytochrome P-450 and $17 a$-hydroxylase cytochrome P-450 in bovine ovarian follicles Journal of Reproduction and Fertility 78 627-638

Sanes JR and Chiu AY (1983) The basal lamina of the neuromuscular junction Cold Spring Harbor Symposia for Quantitative Biology 48 667-678

Schuger L, Skubitz APN, Gilbride K, Mandel R and He L. (1996) Laminin and heparan sulfate proteoglycan mediate epithelial cell polarization in organotypic cultures of embryonic lung cells: evidence implicating involvement of the inner globular region of laminin beta $I$ chain and the heparan sulfate groups of heparan sulfate proteoglycan Developmental Biology 179 264-273

Smith C and MacKay S (1991) Morphological development and fate of the mouse mesonephros Journal of Anatomy 174 171-184

Timpl R and Brow JC (1994) The Laminins Matrix Biology 14 275-281

Timpl R and Dziadek M (1986) Structure, development and molecular pathology of basement membranes International Reviews of Experimental Pathology 29 1-112

van Wezel IL and Rodgers RJ (1996) Morphological characterization of bovine primordial follicles and their environment in vivo. Biology of Reproduction 55 1003-1011 
Walker-Caprioglio HM, Hunter DD, McGuire PG, Little SA and McGuffee LJ (1995) Composition in situ and in vitro of vascular smooth muscle laminin in the rat Cell and Tissue Research 281 187-196

Watt FM (1986) The extracellular matrix and cell shape Trends in Biochemical Science $11482-585$

Wordinger RJ, Rudick VL and Rudick MJ (1983) Immunohistochemical localization of laminin within the mouse ovary Journal of Experimental Zoology $228141-143$

Yoshimura Y, Okamoto T and Tamura T (1991) Localization of fibronectin in the bovine ovary Animal Science and Technology 62 529-532

Yoshinaga-Hirabayashi T, Ishimura K, Fujita H, Kitawaki J and Osawa Y (1990) Immunocytochemical localization of aromatase in immature rat ovaries treated with PMSG and hCG, and in pregnant rat ovaries Histochemistry 93 223-228

Yurchenco PD and Schittny JC (1990) Molecular architecture of basement membranes FASEB Journal 4 1577-1590
Zhao $Y$ and Luck MR (1995) Gene expression and protein distribution of collagen, fibronectin and laminin in bovine follicles and corpora lutea Journal of Reproduction and Fertility $104115-123$

Zhou J, Ding M, Zhao Z and Reeders ST (1994) Complete primary structure of the sixth chain of human basement membrane collagen, $\alpha 6$ (IV) Journal of Biological Chemistry $26913193-13199$

Zlotkin T, Farkash Y and Orly J (1986) Cell-specific expression of immunoreactive cholesterol side-chain cleavage cytochrome P-450 during follicular development in the rat ovary Endocrinology 119 2809-2820

Zoller LC and Weisz J (1978) Identification of cytochrome P-450, and its distribution in the membrana granulosa of the preovulatory follicle, using quantitative cytochemistry Endocrinology 103 310-314

Zoller LC and Weisz J (1979) A quantitative cytochemical study of glucose-6phosphate dehydrogenase and $\Delta 5-3 \beta$-hydroxysteroid dehydrogenase activity in the membrana granulosa of the ovulable type of follicles of the rat Histochemistry 62 125-135 